\title{
La eficacia del tratamiento con ultrasonido en el sindrome del túnel carpiano
}

Ultrasound treatment fot treating the carpal tunnel syndrome : randomised "sham" controlled trial. Ebenbichler GR, Resch KL,Nicolakis P, et. al. British Med. Journal; 1998;316:731-735.

\section{Objetivo}

Determinar la eficacia del tratamiento con ultrasonido en el sindrome del túnel carpiano idiopático leve a moderado.

\section{Diseño}

Estudio clínico randomizado, doble ciego, con seguimiento a seis meses.

\section{Lugar}

Departamento de kinesiología y rehabilitación de la Universidad de Viena, Austria.

\section{Pacientes}

Participaron 45 pacientes con sindrome del túnel carpiano bilateral de origen idiopático, sin tratamiento previo, utilizandose para su diagnóstico criterios clínicos y electromiográficos (EMG).

\section{Intervención}

Se estudiaron ambas muñecas de cada paciente recibiendo una el tratamiento simulado y la otra ultrasonido. Esta asignación fue randomizada. El ultrasonido fue administrado como monoterapia en 20 sesiones de 15 minutos cada una sobre el área del túnel carpiano, siendo las 10 primeras diarias, y las 10 últimas bisemanales. Los pacientes podían recibir analgésicos sin actividad antinflamatoria.

\section{Medición de Resultados Principales}

Se midieron los síntomas de dolor y/o parestesias en cada muñeca, cuantificados en una escala visual colorimétrica, con un extremo de coLor blanco representando "ninguna molestia", y otro extremo de color rojo de "la máxima molestia". La pérdida de la sensibilidad fue valorada con una rueda dentada comparándose con el $5^{\circ}$ dedo de la misma mano. Los signos medidos con el EMG fueron: conducción del nervio motor mediano, latencia y potenciales de acción sensitivos.

Los resultados secundarios fueron la medición de la fuerza con un dinamómetro de uso manual (hand grip), la fuerza en la pinza entre el indice y el pulgar, así como la sensación subjetiva de mejoría del paciente.

\section{Resultados}

Los pacientes con tratamiento activo presentaron una mejoría de los sintomas medidos con las escalas colorimétricas de un $68 \%$ versus $38 \%$ en el grupo placebo al final del tratamiento $(P<0,001$ con RRR del $48 \%)$, y del $74 \%$ versus $20 \%(P<0,001)$ a los 6 meses de seguimiento. La latencia motora distal también disminuyó con el tratamiento activo, y se observaron cambios positivos significativos en la velocidad de conducción de los nervios sensitivos. El hand grip mejoró, como así también la fuerza de la pinza entre los dedos.

No se reportaron efectos adversos con el ultrasonido. El consumo de analgésicos durante el estudio fue bajo.

\section{Conclusiones}

El tratamiento con ultrasonido para el sindrome del túnel carpiano idiopático leve a moderado es efectivo a corto y mediano plazo.

\section{COMENTARIO}

El sindrome del túnel carpiano (STC) es la neuropatía periférica por atrapamiento mas frecuente, siendo la mitad de ellos de origen ocupacional (1). Afecta con mayor frecuencia a mujeres de edad media, y se asocia a otras condiciones tales como DBT, hipotiroidismo y artritis (2).

La compresión del nervio mediano produce la sensación de adormecimiento, parestesias, y dolor en los dedos índice, medio, anular y cara palmar del pulgar, con irradiación proximal, e hipoestesia. Casi en la mitad de los casos es bilateral. El diagnóstico se hace con la clínica, siendo el test de referencia el EMG, el cual sirve además para realizar diagnósticos diferenciales con radiculopatía cervical y neuropatía periférica difusa (1). En los pacientes con síntomas leves e intermitentes, el tratamiento es conservador, con colocación de una valva en posición neutral que impida los movimientos y/o anti-inflamatorios. Las inyecciones locales con corticoides dentro del túnel carpiano mostraron una mejoría del $80 \%$ a corto plazo, disminuyendo al año a un $22 \%$.

El tratamiento quirúrgico se reserva para pacientes mayores de 50 años, con síntomas limitantes durante más de 10 meses, parestesias permanentes y fracaso con el tratamiento conservador. Si bien los resultados iniciales pueden ser exitosos con una mejoría del $80 \%$, el seguimiento a 2 años mostró una recaída de la sintomatología inicial del 57 \% (3). A pesar que el tratamiento con ultrasonido ha demostrado efectividad en patologías como la epicondilitis, no había sido hasta ahora evaluado en el STC(4). Varios puntos refuerzan los hallazgos de este interesante es- tudio. Primero, el ultrasonido fue aplicado mediante una llave simulada, por lo cual los kinesiólogos estaban ciegos al tratamiento efectuado. Segundo, el tratamiento aplicado alternativamente para cada muñeca del mismo paciente, los transforma en sus propios controles, permitiendo discriminar mucho mejor si la mejoría obtenida se debió a la intervención. Tercero, el protocolo explícitamente excluía otras formas de tratamiento no invasivo como los anti-inflamatorios. Cuarto, la mayoría de los tratamientos disminuyen su efectividad a lo largo del tiempo, pero con el ultrasonido la mejoría aumenta a los 6 meses.

Algunas observaciones metodológicas deben ser consideradas antes de aceptar las conclusiones: 1) El reclutamiento de pacientes fue bajo y esto podría obedecer al hecho de que algunos pacientes con dolor rehusen participar en un estudio en el que pueden no recibir tratamiento, y esto puede seleccionar pacientes con menor compromiso sintomático, limitando su validez externa*. 2) La pérdida de pacientes fue muy elevada (33\%). Llama la atención la falta de discusión sobre los datos de estos pacientes ya que, si se aplicara un análisis de sensibilidad ${ }^{*}$ asumiendo falta de respuesta en los que abandonaron, los resultados podrían modificarse al punto de no demostrar eficacia de la intervención. 3)El tiempo de seguimiento fue escaso en comparación con la mayor parte de los estudios en que el seguimiento fue a dos años. De confirmarse los hallazgos del estudio resultará sin duda interesante poder recurrir a una alternativa terapéutica no invasiva, de bajo costo y eficaz para el tratamiento de este sindrome.

*Ver Glosario 